\title{
A study on the Computing Framework of Cloud Intelligence Business Platforms
}

\author{
Feiqi Wang, Jian Zhang \\ School of Beijing University of Technology 100020 China; \\ 15210026808@163.com
}

Keywords: SOA cloud computing; service composition; distributed system

\begin{abstract}
With the development and application of business management information system, businesss own much more production and management softwares. Traditional computer labs can not deploy these application softwares and provide data sharing operations at the same time. In this paper, a cloud intelligence business platform based on the theory of framework and business model of SOA and cloud computing is discussed. A good software support will be achieved by virtue of ideas of hierarchical design of cloud platforms and virtualization technology to extend business hardware resources.
\end{abstract}

\section{Introduction}

Nowadays, the construction of business information has become a trend which can not be halted. More and more applications have taken place of manual management, greatly improving the efficiency of all departments of the business. On the other hand, with the growth of business application systems, the data and information stored in various business systems are also increasing. System construction is independent. More and more funds are invested. However, problems also exist. There are four problems needing to be resolved: first, because the system data is independent, information can not be effectively shared inside the businesses, resulting in " lonely islands of information"; second, the information system did not form a unified standard, causing high cost of systems; thirdly, each application system is deployed on a server by itself, so the server utilization rate is low, leading to waste of resources; fourth, each system has a different set of user name and passwords. But business users need to remember user name and password corresponding to the system, bringng about a lot of inconvenience.

In order to solve the above problems, all major businesss actively explore information construction. With the advent of the cloud platform, problems can be dealt with by its technologies.

\section{Relevant concepts}

\subsection{SOA}

SOA, which was firstly introduced by Gartner in a report of 1996, is a distributed application construction approach focusing on "service, loose coupling, dynamical optimization, and reuse". During the past a decade of development, it has become the trend of constructing an integrated distributed information system in an open and heterogeneous network environment. SOA has many advantages, such as loose coupling, interoperability, and large-scale reuse, integration, flexibility to bring new impetus and opportunity for the establishment, integration and maintenance of software system, especially the innovation and development of Internet software industry.

\subsection{Cloud computing}

There are many different definitions of cloud computing. In January, 2011, NIST defined cloud computing as a usage pattern of IT resources to provide ubiquitous, convenient, on-deman online access to shared and configurable computing resources (such as network, server, storage, application and services). 


\subsection{SOA and cloud computing integration research}

According to David S. Linthumum, a U.S. author for Cloud Computing and SOA, published in 2011, " if cloud computing brings great value to SOA, cloud computing can be considered as a exended resource for SOA to the cloud platforms. It provides the IT resources that SOA can use on demand, and SOA can be used as a key technology to realize cloud computing." SOA addresses business requirements while cloud computing more solves technology to IT infrastructure unit. SOA is the business system to respond flexibly to changes in business needs, while cloud computing enables the hardware environment to respond flexibly to business systems. Therefore, the combination of both helps to improve the flexibility of business system modification and cost scalability.

\section{Cloud intelligence business platform system framework design}

In this paper, the cloud intelligence business platform is one with commercial nature. The platform offers services to three different types of users, including company employees, business managers and third-party agencies. Different types of users clients publish and obtain a variety of required business information servicesin the business cloud on the platform, including daily attendance, daily process approval, daily work reports, business management reports and other services. The business cloud platform is verified by implementing a prototype service-oriented C / S architecture cloud service platform. Clients utilizes the mobile or Web side to get services. All data generated by the server maintenance service and a series of service components publish a variety of business information services through the service component. Through the prototype construction of the above-mentioned business cloud service ecosystem, a viable solution to the problem of China's smart business has been explored.

From the level of cloud computing services and the scope, computing framework of high-performance cloud intelligence business is divided into three levels, consisting of cloud intelligence business platform infrastructure layer (IAAS), cloud intelligence business cloud service platform layer (PAAS), cloud intelligence business application and software system layer (SAAS).

\subsection{IAAS layer}
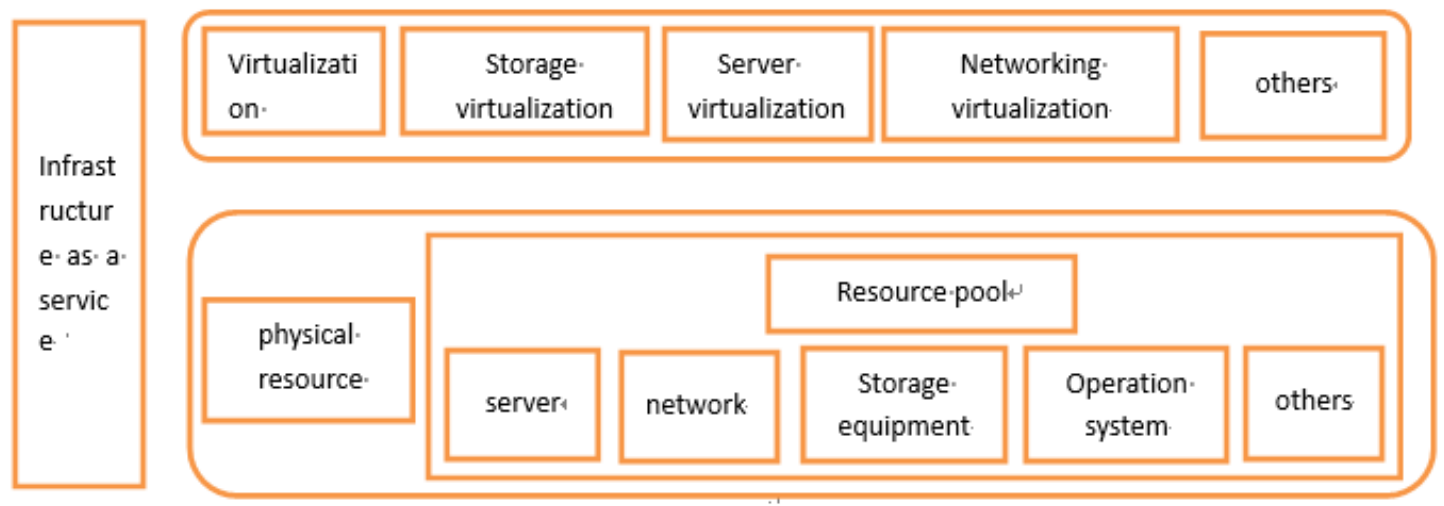

Fig.1 Computing framework of IAAS layer

Cloud Intelligence business Platform Infrastructure Layer (IAAS): The infrastructure here refers to IT facilities including computers, storage, networking, and other related software and hardware facilities. The main function of the IAAS layer is that renters input their own number of users. The cloud-based education platform IAAS cloud service providers allocate IT basic resource services required by the renters. The IAAS includes a virtualization platform and a resourceless resource layer.

Physical resources layer: includes servers, networks, storage devices, operating systems. These resources are logically stored together as a large pool of resources.

Storage Virtualization: allocates to virtual machines by dividing a large storage space into multiple smaller storage spaces. Storage virtualization is for storage and isolation of resources based 
on the file as a unit. This file is not a specific file seen in the virtual machine, but a large file used on the physical machine to emulate the virtual machine's hard disk. Access to the hard disk address is an offset to the file.

Server virtualization: These servers are firstly virtualized into multiple servers. These servers can also be called virtual machines. We deploy applications that occupy less resources on virtual machines. These applications are like deploying Independent server, the virtual machine can be divided in accordance with the amount of resources

Network Virtualization: When multiple virtual machines are created on a physical machine, each virtual machine needs an independent network configuration so that they can handle all kinds of network connections like a physical machine. However, at this time, there is still only one network card on the physical machine and networking virtualization. Can be formed if multiple virtual machines can smoothly access to network.

\subsection{PAAS}

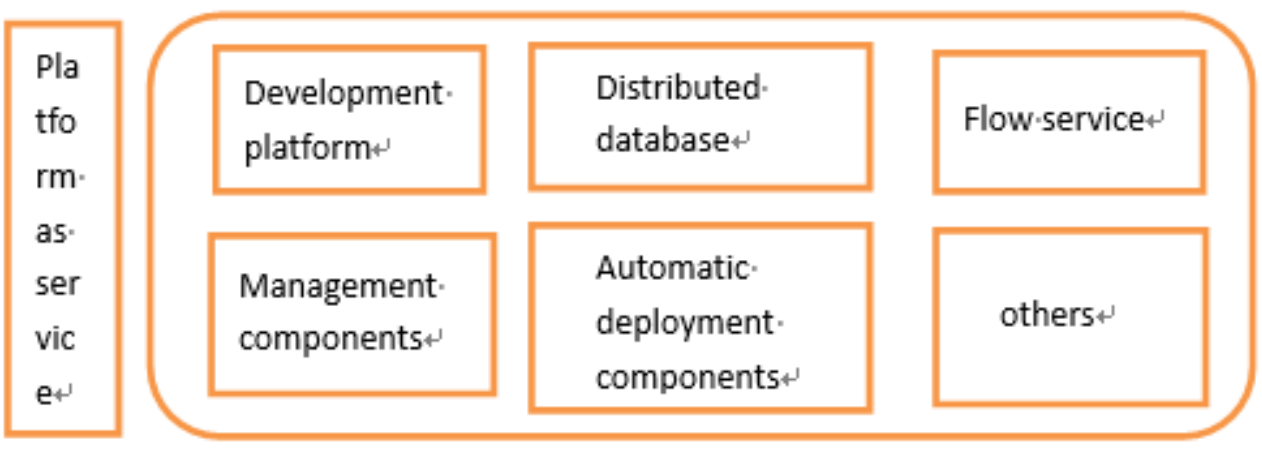

Fig.2 Cloud computing framework of PAAS layer

Cloud Intelligence business Platform Layer (PAAS): A complete PaaS platform needs to achieve IT resources integration, effective management, improve utilization, allocation of resources on demand and measurement; unified development and testing, operating platform, the development of test environment and production environment linkage, seamless upgrading of platforms and applications, automated delivery, reduction of manual intervention, quick deployment / flexible expansion, standardized operation and maintenance system, etc. IaaS, PaaS and SaaS interfaces are integrated into a unified cloud management console for unified management

The main users of PaaS are software development, deployment and system administrators. Therefore, full life-cycle support for applications include:

(a) Application development and deployment of operating environment: Distributed operating environment, operating system, language execution environment and application server support development, testing and deployment of SDK, IDE and other applications.

(b) Application Services Component Pooling and Management: Provides public services such as databases, message queues, caches, and various types of data storage in API form;

(c) Services, applications and resource management: service and application deployment, updating and deletion, resource monitoring, management, and metering applications.

On the other hand, application developers can do other things on the development platforms:

(a) develop, operate and manage their application systems without giving care for the operation of underlying hardware devices and software resources;

(b) You can also automatically expend the underlying resources as needed to automatically configure and expand the technology and required storage resources for your application.

\subsection{SAAS layer}

Software as a service is a SaaS-based software delivery model at the top of the cloud platform, providing software services with businesss, including portals, application services, systems management, and data centers. 


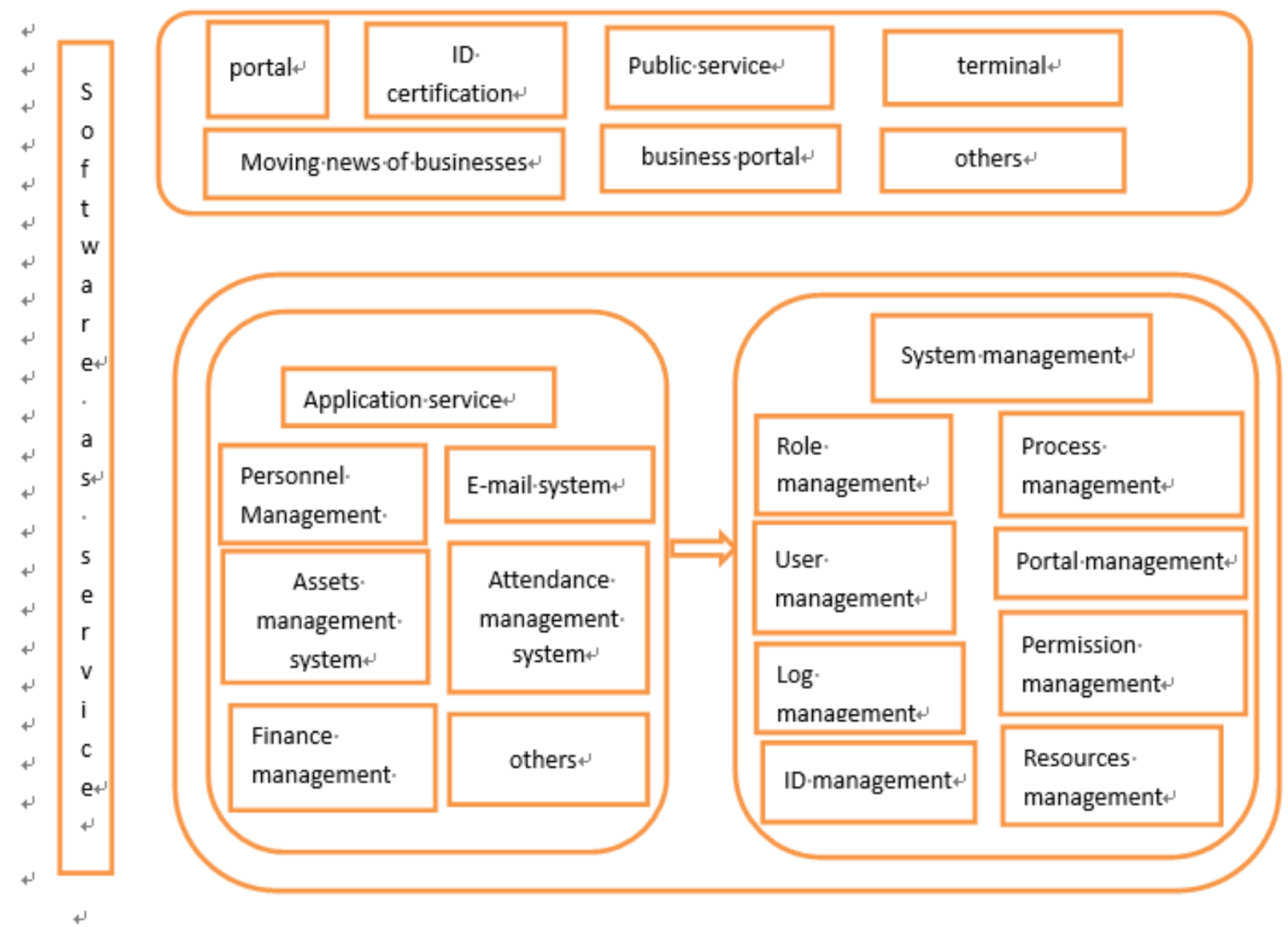

Fig.3 Cloud computing framework of SAAS layer

(1) Business Portals

Portal is the main interface of the business information website, mainly including business news, bulletins, announcements and business moments and other content, while the internal application of the business entrance page is located in the portal site of the application system-the business Information management window. On the other hand, it also serves as the main channel for business information collection and release. Considering the actual needs of businesss, we can divide the business portal into a unified identity portal, interface integration, public services, terminal equipment, business dynamics, cooperation Partners and business users.

(2) Unified certification

Unified identity certification is the identity authentication service when entering the business application system. There are many application systems in the business. The unified identity ceritification allows a single entry of the real system. A user only needs a group of user name and password to access all the systems within his / her authority.

(3) Business application system

Business application system is related to a variety of business applications business systems. These businesses include business finance, personnel, production and logistics. The major systems keep a line with all business. Application system in the development process should adopt unified norms and standards. Application system should not only maintain relative independence, but also have a certain degree of coupling. There are many application systems in an business, so an overall planning is needed. Each application system can develop and deploy in different stages. In the course of development, it is required to follow the standards of business informationization and system integration standards. 


\section{Business cloud platform multi-renter model design}

At present, there are mainly three modes for implementing multi-renters technologies: physical isolation, virtualized isolation and application isolation.

Physical isolation mode is reflected in one to one. Each renter is configured with independent resources, including software resources and hardware resources to achieve isolation through exclusive resources. For the user who need some personalized design, thay are carried out in their own exclusive Resources, carried out on other users without impacting other users. This mode is similar to the traditional software delivery mode, only adding users' configuration with advantage of high security level, which is easy to implement, but the disadvantage of this approach is also very obvious with low level of resource sharing, resulting in low resource utilization. The cost of hardware and software for users will be very high, so this method is suitable for large and medium-sized businesss, but not for some small businesses.

Virtualization isolation is accomplished by configuring virtual machines. The basic principle is to configure multiple virtual machines on a specific physical machine to virtualize several systems so that each renters uses a system that uses virtual machine technology to isolate the operating system level between the renters. Compared to the previous way, this kind of physical way has been improved on the utilization of resources. But when there are more and more users, physical machines will have to virtualize a lot of systems. Scheduling algorithm will be very complicated, while the cpu, memory resources of physical machine are limited, too many virtual machines will certainly affect performance of the system.

Three application isolation modes are mainly single-instance multi-tenancy mode. Multiple renterss are served through the sharing of one instance. This mode meets renterss' personalized needs through application-level renters customization and ensures that renterss can share storage system and hardware-level resources by designing multi-renters data storage and performance assurance.. One of the major advantages of this isolation mode is great improvement of shared resources. The cost of hardware and software is reduced, but the corresponding degree of segregation among renterss also drops a lot. Therefore, this model is more suitable for small and medium businesss renters.

The business cloud platform, studied in this paper, uses a multi-tenancy management model based on application isolation mode. Logically, renters segregation is realized by sharing instances. Renters management model is divided into registration, renting and purchase, customization, billing. When a renter registers on this platform, multiple user attributes can be created at the same time. The concept of renters ID and user ID is introduced here to achieve isolation between data tuples, maximize resource sharing, and improve resource usage efficiency.

When renters rent a platform service, different approaches have different pricing strategies. The platform allocates servers, infrastructure hardware, network bandwidth, service components, data storage space and other resources according to the number of subscribers. For each resource, it has a different billing model. The platform mainly has three kinds of billing modes: the length of rental billing, charging by the number of rentals and charging by flow data. Whenever a renter buys one or more services, the system generates a lease record for the renters to record the renters's charging policy for the leased resources selection and data such as the service term and number of services.

Renters apply for personalized service on this platform. In this case, the service is mainly analyzed according to the renters's business needs. The original service of the platform is called and combined to generate a new unique service suitable for renters. For this new service, in principle, only renters who apply for customization are allowed to use the service, which means that other renters who do not customize this service will not be entitled to invoke the service.

The business cloud platform adopts a water-flow charging mode and summarizes a file log for generating reports by using resource of renters and bills. There are different billing standards for different rental mode. Detailed account in the generated bill can be offered to renters. 


\section{Multi-renters database storage model design}

In the SaaS application, multi-renters data storage framework mainly has three modes: independent database, shared data database, isolated data, shared database, shared data framework.

Independent database approach means that a renters has a database. This model is characterized by high security, highest data isolation. Once there is a problem, the data can also be restored immediately. However, the problem with this model islisted as follows: large installation volume and relatively high maintenance costs. Specific model structure shows as follows:
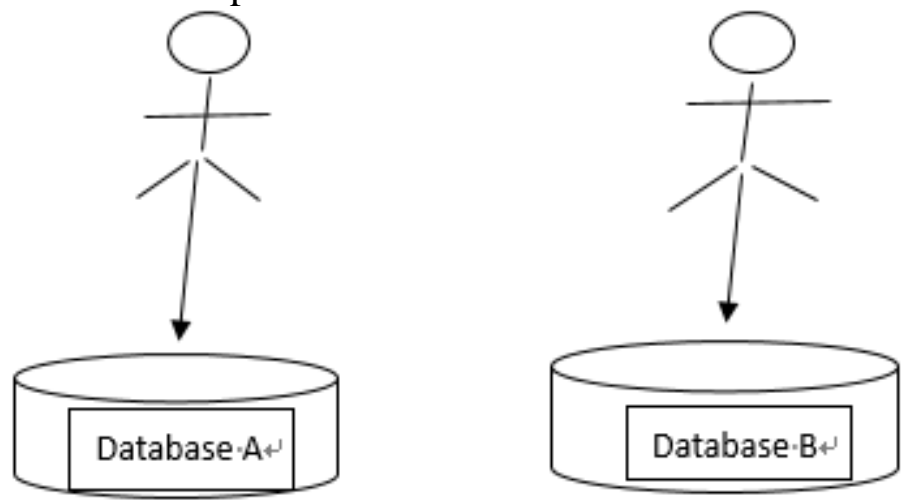

Shared data database and isolated data framework refers to that multiple users share a database, but a renter occupies only one framework. This mode allows multiple renters to use it. However, the data isolation is more difficult. At the same time, it is difficult to restore data. Specific structure is shown below:

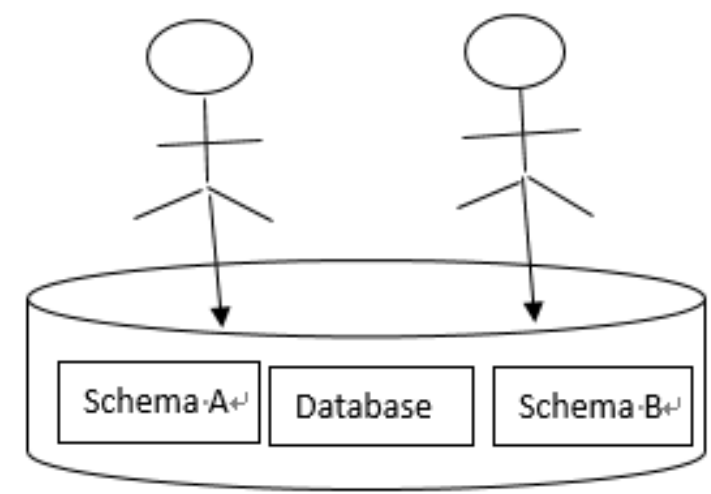

Shared database and shared data architecture allow multiple renters to share a database and aframework. This model means that ID number can be added in different renters table. A typical disadvantage of this mode is the low security, but with low cost. To solve this problem, a T_renters data table is added in the database table, recording the ID information of each renter. An ID field area is added in the user tables of each business to perform different data Isolation. After users log in the system, they can search and filter the data through extra renters_ID added to achieve the isolation and protection of different renters data.

According to the actual situation, the platform selects the database storage framework of "shared database and shared data architecture mode" and designs a distributed data storage system - the HDFS storage system. It stores all the generated files in the HDFS file storage system, and Address storage is stored to HBase file storage system, to avoid frequent mergers and segmentation for improvement data processing performance. The goal of designing distributed processing system is to utilize MapReduce parallel algorithm to improve the ability of system data processing and computing and the efficiency of system operation. 


\section{Summary}

The development and maturity of cloud computing has brought a series of cross-industry joint models. It is practical and certain to design and implement the intelligent business cloud platform by using cloud computing cloud services technology. Based on SOA technology design system framework and cloud computing technology, much business information will be collected. A large number of users on the platform can be taken on demand to meet their different business needs. It will be more widespread in the future of industrial applications.

\section{References}

[1]. Yu Chaoxin, TanWei-ji, Zhang Jing-xian. A study on Multi-Tenancy Management Technology Based on Distributed Cloud Computing. Data Communications, 2016, (4): 7-11.

[2]. Zhang Ting .J2EE Framework in Intelligent Community Management System Application Research and Design [D]. Shaanxi University of Science and Technology, 2016.

[3]. Vic (J.R.) Winkler. Securing the Cloud: Cloud Computer Security Techniques and Tacties. 2013

[4]. Chen Xiaohua. Cloud-oriented Manufacturing Platform for SMEs [J]. Modern Manufacturing Technology and Equipment, 2014 (3): 37-39.

[5]. Ma Mingli. Cloud Foundry-Based Integrated Service Platform for Research and Implementation [D]. Dalian: Dalian University of Technology .2014

[6]. Shu Weiguo, Zhang Shuguang. White Paper on the Development of business Cloud Construction [R]. Hefei: Economic and Information Technology Commission of Anhui Province, 2016.

[7]. Journal of Jiangxi Normal University (Natural Sciences Edition), 2014 (04): 1.37. [7] Huang Yong, Zhou Yong, Liu Zhiping.A New Generation of College Test Database System Based on SOA [J] .Journal of Jiangxi Normal University,

[8]. Wu Changyu, Li Yunsong, Liu Qing, et al.Design of IOT Application Framework Based on Microservices Architecture [J] .Journal of Suzhou University, 2015 (07): 92-96.

[9]. Wang Lei. Microservices Architecture and Practice [M]. Beijing: Publishing House of Electronics Industry, 2015.

[10].PATERSON D, FOX, et al. AAA Software Engineering: Cloud Computing Era of Agile Development [M]. Tsinghua University Press, 2015.

[11].Zhang Xuan, Ji Zhiyi. Construction of business-Class Mobile Office Platform [J]. Information Technology and Standardization, 2015 (07): 32-34,54.

[12].Li Guiying, Ma Li.Study on Autonomous Mobile Learning Model Based on Cloud Education Platform [J]. Modern Education Technology, 2011, (11) 